\title{
Enhancement of radiosensitivity by inhibition of c-Jun N-terminal kinase activity in a Lewis lung carcinoma-bearing subcutaneous tumor mouse model
}

\author{
CHUN-HAO LI ${ }^{1}$, SA-HOE LIM ${ }^{1}$, HYANG-HWA RYU ${ }^{1}$, KYUNG-SUB MOON ${ }^{2}$, \\ TAE-YOUNG JUNG ${ }^{2}$ and SHIN JUNG ${ }^{1,2}$ \\ ${ }^{1}$ Brain Tumor Research Laboratory, Chonnam National University Research Institute of Medical Sciences, \\ Chonnam National University Medical School and Chonnam National University Hwasun Hospital; \\ ${ }^{2}$ Department of Neurosurgery, Chonnam National University Medical School and Chonnam National \\ University Hwasun Hospital, Hwasun, Jeollanam-do 519-763, Republic of Korea
}

Received July 7, 2016; Accepted August 26, 2016

DOI: $10.3892 /$ or.2016.5204

\begin{abstract}
Stereotactic radiosurgery has been recognized as an effective treatment approach for metastatic brain tumors. By increasing the sensitivity of the tumor to radiation and decreasing the marginal dose, it is possible to improve therapeutic efficacy and decrease side-effects. In radiation-induced cells, c-Jun N-terminal kinase (JNK) signaling mediates the phosphorylation of $\mathrm{H} 2 \mathrm{AX}$, which indicates DNA damage sensitivity and modulates the effect of radiation. Lewis lung cancer (LLC) and breast cancer (4T1) cells were irradiated with a Gamma Knife in cell culture tubes. To evaluate the relationship between radiosensitivity and JNK activity, clonogenic assay was performed. DNA damage response was estimated by $\gamma \mathrm{H} 2 \mathrm{AX}$ focus formation assay and apoptosis-related protein levels were assessed by western blotting. The mice were subcutaneously inoculated with LLC cells, and irradiated concomitantly with JNK inhibitor treatment. The effect of the JNK inhibitor was investigated by tumor volumetry and immunohistochemistry. $\gamma \mathrm{H} 2 \mathrm{AX}$ expression, which mediates repair of radiation-induced DNA damage, was reduced in the cancer cell group pretreated with the JNK inhibitor. This finding shows that JNK inhibition may increase the radiosensitivity in radiated lung and breast cancer cells. For the in vivo study, irradiated tumor growth was significantly delayed in the JNK inhibitor-treated mouse group. Blockade
\end{abstract}

Correspondence to: Dr Shin Jung, Department of Biomedical Sciences, Brain Tumor Research Laboratory, Chonnam National University Research Institute of Medical Sciences, Chonnam National University Medical School and Chonnam National University Hwasun Hospital, Hwasun, Jeollanam-do 519-763, Republic of Korea

E-mail: sjung@jnu.ac.kr

Key words: JNK, histone $\mathrm{H} 2 \mathrm{AX}$, radiosurgery, radiotherapy, apoptosis of JNK signaling decreased $\gamma \mathrm{H} 2 \mathrm{AX}$ expression and increased apoptosis in the radiation-induced cancer cells. JNK inhibitor may be useful for enhancing the radiosensitivity of lung and breast cancer cells and improving the treatment efficacy of radiosurgical approaches for metastatic brain tumors.

\section{Introduction}

With the development of medical imaging technology and molecular diagnostics, metastatic brain tumors are being detected and treated at earlier stages. The most common treatments for metastatic brain tumors include surgery, chemotherapy and radiotherapy. Radiosurgery is frequently used in patients with metastatic brain tumors. However, when a tumor is relatively large, developed in the brain stem, or close to important nerves such as the optic nerve, it cannot be treated with a high dose of radiation. In these situations, it is imperative to increase the radiosensitivity of cancer cells in order to produce the desired outcome with fewer doses of radiation. Therefore, it is essential to develop radiosensitizers which are non-toxic, effective and molecularly targeted.

The JNK pathway is one subtype of MAP kinase signaling that is activated primarily by cytokines and exposure to environmental stress (1). JNK participates in every type of cellular response, including apoptosis $(2,3)$. JNK also participates in the phosphorylation of $\mathrm{H} 2 \mathrm{AX}$ after radiation (4). Based on this finding, Yue et al reported that inhibition of JNK activity enhanced radiosensitivity and apoptosis in vestibular schwannoma (5). Accumulating evidence has demonstrated the important role of JNK in the process of DNA repair after radiation. However, to the best of our knowledge, no study has reported the effects of JNK small-molecule inhibitor SP600125 on lung and breast cancer cells treated with radiotherapy. On the basis of its efficacy in vestibular schwannoma, it is plausible that inhibition of JNK activity could also increase the radiosensitivity of lung and breast cancer cells.

Currently, the JNK inhibitor, PGL5001, is used to treat inflammatory endometriosis, and has undergone a phase II clinical trial (clinicaltrials.gov ID NCT01630252). The results 
of that study indicated that the JNK inhibitor has the potential to be useful in the treatment of various clinical conditions. The JNK small-molecule inhibitor SP600125 (anthra[1,9-cd] pyrazol-6(2H)-one) specifically inhibited phosphorylation of c-Jun $(6,7)$. In the present study, we investigated the effects of the JNK inhibitor SP600125 in combination with radiation in vitro and in vivo. Our results demonstrated that JNK inhibition increased the radiosensitivity in lung and breast cancer cell lines. Overall, the present study showed that the combination of SP600125 with radiotherapy appears to have promising antitumor activity, and provides therapeutic benefits in the clinical setting for metastatic brain tumor.

\section{Materials and methods}

Cell cultures and treatment conditions. The murine Lewis lung cancer (LLC) and mammary carcinoma (4T1) cell lines were obtained from the American Type Culture Collection (ATCC; Manassas, VA, USA). The cell lines were cultured in Dulbecco's modified Eagle's medium (DMEM) supplemented with $10 \%$ fetal bovine serum (FBS) (both from Gibco-BLR, Gaithersburg, $\mathrm{MD}, \mathrm{USA})$ at $37^{\circ} \mathrm{C}$ in a $5 \% \mathrm{CO}_{2} / 95 \%$ air incubator. SP600125 was kindly provided by Sigma (St. Louis, MO, USA), constituted in dimethyl sulfoxide (DMSO) (10 mM), and stored at $-80^{\circ} \mathrm{C}$. Cellular exposure to SP600125 was performed $2 \mathrm{~h}$ before irradiation with Gamma Knife Perfexion.

MTT assay. The toxicity of SP600125 was monitored using the 3-(4,5-dimethylthiazol-2-yl)-2,5-diphenyltertazolium bromide (MTT) assay. The cells $\left(10^{3}\right.$ cells/well $)$ were seeded into 96-well plates and cultured under standard conditions $(10 \%$ FBS at $37^{\circ} \mathrm{C}$ and $5 \% \mathrm{CO}_{2} / 95 \%$ air). At every $24 \mathrm{~h}$, an aliquot of $10 \mu \mathrm{l} \mathrm{MTT} \mathrm{[5} \mathrm{mg/ml} \mathrm{dissolved} \mathrm{in} \mathrm{phosphate-buffered} \mathrm{saline}$ (PBS)] was added to each well. The medium was removed from each well after $2 \mathrm{~h}$. MTT formazan was solubilized in $200 \mu \mathrm{l}$ DMSO. Optical density was read at $570 \mathrm{~nm}$.

Clonogenic assay. For the colony formation assay, LLC and $4 \mathrm{~T} 1$ cell lines were plated in 6-well culture dishes $\left(10^{2}-1.4 \times 10^{4}\right.$ cells/well $)$ and exposed to compound and $\gamma$-irradiation followed by incubation at $37^{\circ} \mathrm{C}$ for 8-10 days. Cells were fixed in methanol for $5 \mathrm{~min}$ and stained with toluidine blue $(0.1 \%$; Sigma) for $15 \mathrm{~min}$. The number of colonies containing at least 50 cells were counted. The surviving fraction was calculated as the number of colonies divided by the number of cells that were initially plated. Dose enhancement factor (DEF) was calculated at the $10 \%$ survival level. D0.1 was defined as the quotient of D0.1 RT/D0.1 RT + drug. A $\mathrm{DEF}>1$ indicates that the drug is functioning as a radiosensitizer.

Western blotting. Western blot analysis was carried out as previously described $(8,9)$. Whole-cell lysates were isolated by RIPA buffer. Lysates were separated by 8-12\% SDS-PAGE and transferred onto polyvinylidene difluoride (PVDF) membranes (Pall Corporation, Port Washington, NY, USA). The membranes were probed with antibodies against GAPDH, P-JNK, BAX, P-Bcl-2, cleaved caspase-3, cleaved PARP and phospho-histone H2AX (Cell Signaling Technology, Danvers, MA, USA) overnight at $4^{\circ} \mathrm{C}$. Bound secondary antibody was visualized by goat anti-rabbit antibody (Jackson Immunoresearch Laboratory, West Grove, PA, USA). The imaging was obtained using an LAS-4000 instrument (Fuji, Tokyo, Japan). GAPDH was used as an internal control.

Immunocytochemistry. The cells were plated at a concentration of $1.2 \times 10^{5}$ cells/well into a 8 -well chamber slide (Labtek Pty Ltd., Brendale, Australia). After $6 \mathrm{~h}$, the slide was fixed for 20 min with $4 \%$ paraformaldehyde, washed with PBS, and the cells were permeabilized for 15 min with $0.8 \%$ Triton-X 100 . The cells were blocked with $5 \%$ goat serum and $0.8 \%$ Triton-X 100 for $30 \mathrm{~min}$ followed by three 5-min PBS washes. The rabbit monoclonal anti-phosphorylated Ser139 histone H2AX (1:300; Cell Signaling Technology) antibody was added and incubated overnight at $4^{\circ} \mathrm{C}$. The cells were exposed to goat anti-rabbit secondary antibody (1:400 in PBS) conjugated with Alexa Fluor 488 or 568 (1:400; Molecular Probes, Sunnyvale, CA, USA). Nuclei were stained with 4',6-diamidino-2-phenylindole (DAPI). Images were captured with an Olympus FV10-ASW confocal laser scanning biological microscope system. The specific immunofluorescence signal in the nucleus was observed using Alexa Fluor 488 or 568 and DAPI fluorescence filters (10). Quantification of the $\gamma \mathrm{H} 2 \mathrm{AX}$ foci was conducted, such that cells were counted when they contained $>5$ foci. At least 50 cells were counted.

Animal studies. Female 7- to 8-week-old C57BL/6 mice were obtained from Orient Co. (Seongnam, Korea). LLC cells $\left(5 \times 10^{5}\right)$ in $50 \mu 1$ of PBS were subcutaneously inoculated into the backside and both hind limbs of mice $(n=5)$ and allowed to grow for 10-11 days. To assess the toxicity of SP600125, right hind limb tumors were directly injected with DMSO (dissolved in PBS), and left hind limb tumors were directly injected with $5 \mu \mathrm{M}$ SP600125 (dissolved in DMSO and PBS) twice daily to termination. For the radiosensitivity study, backside tumors (control) and right hind limb tumors were directly injected with DMSO (dissolved in PBS), and left hind limb tumors were directly injected with $5 \mu \mathrm{M}$ SP600125 (dissolved in DMSO and PBS) $6 \mathrm{~h}$ before localized irradiation. The drugs were injected twice daily to termination. Both hind limbs were irradiated with a fractionated schedule $(3 \times 3.5 \mathrm{~Gy}$, total $8 \mathrm{~Gy})$. Tumors were measured with calipers and the volume was calculated with the formula: Volume $(\mathrm{V})=$ length (a) $\mathrm{x}$ width (b) $\mathrm{x}$ width (b) x 0.5. Radiation was delivered with a 6-MV X-ray linear accelerator (Clinac 21EX; Varian Inc., Palo Alto, CA, USA).

Immunohistochemistry. Tumor tissues were removed 10 days after radiotherapy. The paraffin-embedded specimens were dewaxed in xylene and subjected to heat-mediated antigen retrieval in target retrieval solution ( $\mathrm{pH}$ 9.0; Dako, Carpinteria, CA, USA). Endogenous peroxidase activity was blocked by $3 \% \mathrm{H}_{2} \mathrm{O}_{2}$, and the sections were incubated with $3 \%$ bovine serum albumin (Sigma) to block any non-specific binding. Anti-cleaved caspase-3 (Cell Signaling Technology) was added at $4^{\circ} \mathrm{C}$ overnight, and then the secondary antibody (Dako) was added, and samples were incubated at room temperature for $1 \mathrm{~h}$. The tissue sections were detected using DAB (Dako). Nuclei were stained with Harris hematoxylin (ScyTek, Logan, UT, USA). 
A LLC

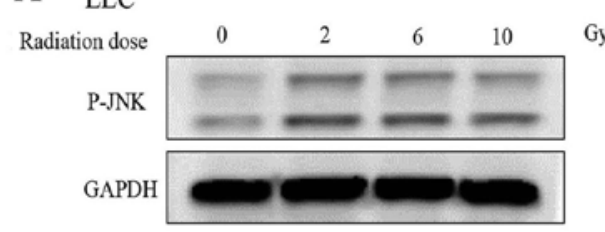

B

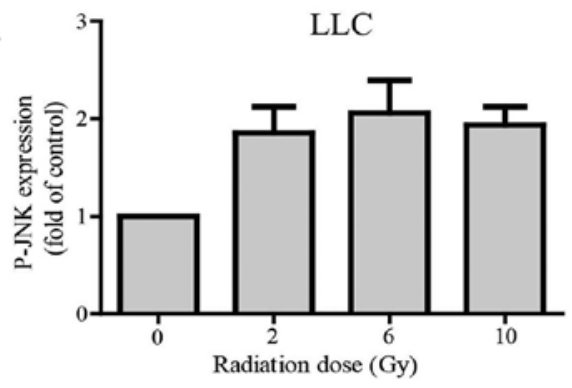

4T1
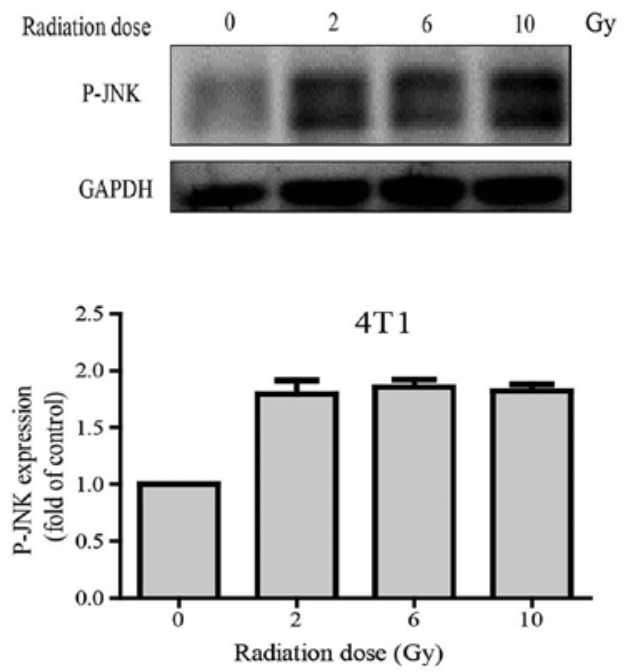

Figure 1. Effects of radiation on P-JNK expression in LLC and 4T1 cells. The cells were exposed 0, 2, 6 or 10 Gy radiation, as indicated. (A) Cells were harvested for western blot analysis $24 \mathrm{~h}$ after radiation. (B) Histograms showing P-JNK expression levels relative to the control. GAPDH was used as the internal control. Bars indicate $\pm \mathrm{SD}$; columns, mean.

A

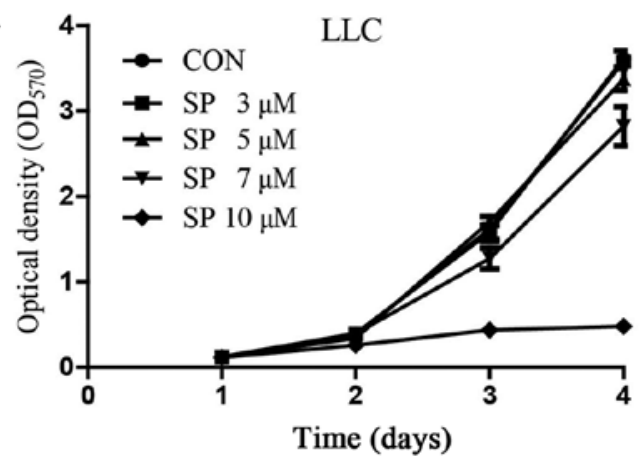

B

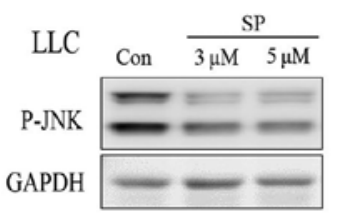

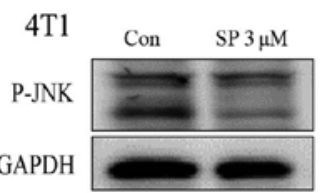

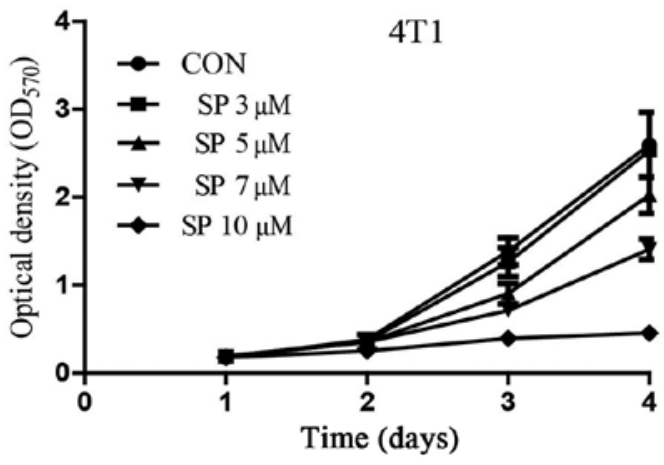

$\mathrm{C}$

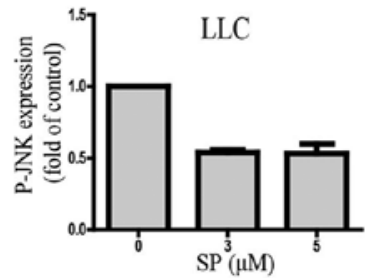

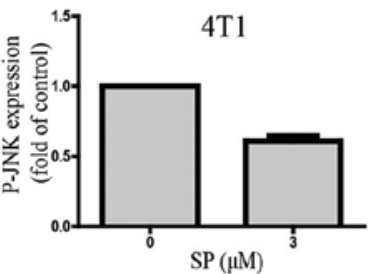

Figure 2. Responses of LLC and 4T1 cell lines to SP600125. (A) Toxicity of SP600125 was determined with the MTT assay in the LLC and 4T1 cells. MTT cell viability assays were performed on days 1-4 after treatment with the indicated concentrations of SP600125. Statistical analysis revealed that the $\mathrm{IC}_{50}$ was $8.14 \mu \mathrm{M}$ and the $\mathrm{IC}_{5}$ was $5.10 \mu \mathrm{M}$ for LLC cells; and the $\mathrm{IC}_{50}$ was $7.37 \mu \mathrm{M}$ and the $\mathrm{IC}_{5}$ was $3.04 \mu \mathrm{M}$ for $4 \mathrm{~T} 1$ cells. (B) SP600125 suppressed JNK signaling at a concentration of $5 \mu \mathrm{M}$ in LLC cells and $3 \mu \mathrm{M}$ in $4 \mathrm{~T} 1$ cells. (C) Histogram showing P-JNK expression levels compared to the control. GAPDH was used as the internal control. Bars indicate $\pm \mathrm{SD}$; columns, mean.

Statistical analyses. Data are presented as mean \pm SD. Between-group comparisons were performed using the Student's t-test. The paired t-test was used to compare the volume of two tumors in one individual. A value of $\mathrm{P}<0.05$ was considered to indicate a statistically significant result.

\section{Results}

Responses of the LLC and $4 T 1$ cell lines to $\gamma$-irradiation and SP600125. Western blot data showed that P-JNK expression was significantly increased in the radiation group compared with that noted in the control group in both the LLC and 4T1 cell lines. Nevertheless, P-JNK expression was independent of the dose of $\gamma$-irradiation (2, 6 and $10 \mathrm{~Gy}$ ) (Fig. 1A and B). In the presence of the JNK inhibitor SP600125, JNK signaling was found to be specifically blocked in other cells $(11,12)$. SP600125 was used in the present study. MTT assay results showed that optical density dose-dependently decreased after SP600125 treatment in the LLC and 4T1 cells. The cells barely grew in the culture medium containing $10 \mu \mathrm{M}$ 
A
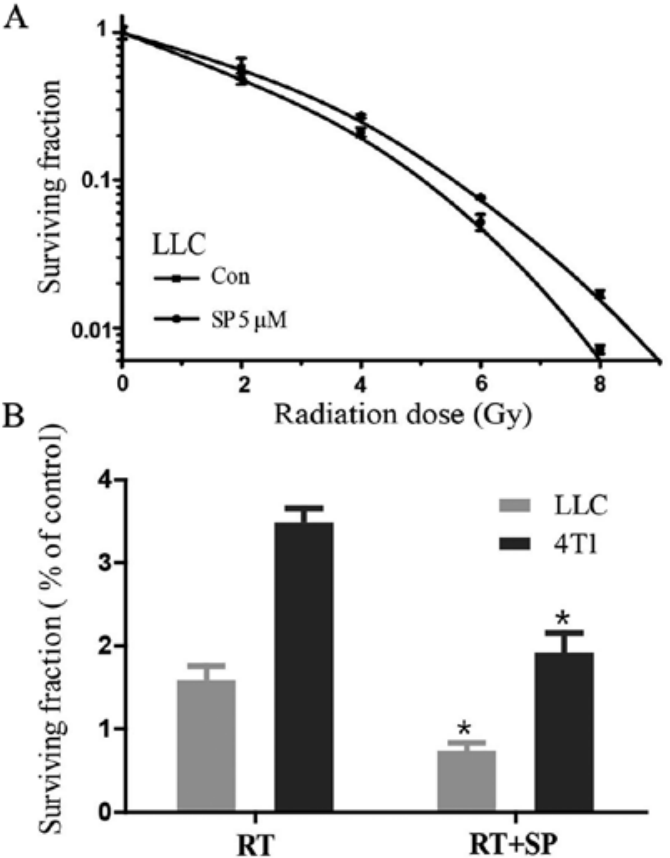

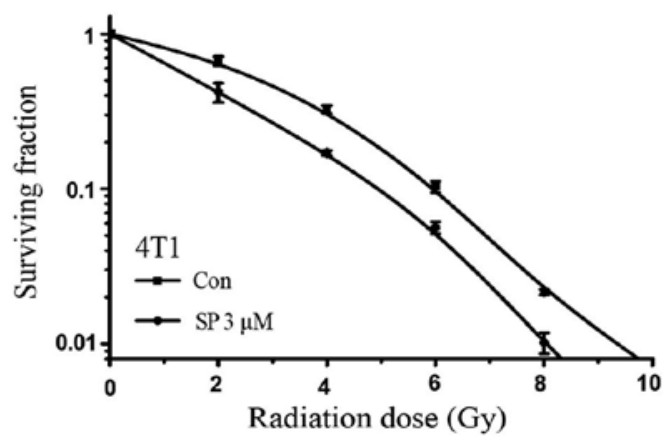

Figure 3. Inhibition of JNK signaling increases radiosensitivity in vitro. Cells were pretreated with a JNK inhibitor for $2 \mathrm{~h}$ before being exposed to graded doses of $\gamma$-irradiation. (A) The effects of SP600125 on the radiosensitivity of LLC (left) and 4T1 (right) cells. (B) Detailed analysis of the clonogenic surviving fraction for RT \pm SP in LLC and 4T1 cells. All experiments were independently performed at least three times. Effects are normalized to control. Bars indicate \pm SD; columns, mean; SP, SP600125 $(5 \mu \mathrm{M}$ for LLC; $3 \mu \mathrm{M}$ for 4T1); RT, radiation ( $\gamma$-irradiation $8 \mathrm{~Gy}$ ); ${ }^{*} \mathrm{P}<0.05$ vs. the RT.
Table I. Dose enhancement factor (DEF) in LLC and 4T1 cells.

\begin{tabular}{llcc}
\hline Cells & Treatment & D0.1 (Gy) & DEF \\
\hline LLC & RT & 5.56 & \\
& RT + SP & 5.02 & 1.11 \\
4 T1 & RT & 5.93 & \\
& RT + SP & 4.89 & 1.21 \\
\hline
\end{tabular}

A DEF $>1$ indicates that the drug functions as a radiosensitizer. LLC, Lewis lung cancer; SP, SP600125; RT, radiation ( $\gamma$-irradiation).

SP600125 (Fig. 2A). The $\mathrm{IC}_{5}$ of SP600125 was obtained by statistical analysis. The $\mathrm{IC}_{50}$ was $8.14 \mu \mathrm{M}$ and the $\mathrm{IC}_{5}$ was $5.10 \mu \mathrm{M}$ for LLC, and the $\mathrm{IC}_{50}$ was $7.37 \mu \mathrm{M}$ and the $\mathrm{IC}_{5}$ was $3.04 \mu \mathrm{M}$ for $4 \mathrm{~T} 1$. For the subsequent clonogenic assay, doses of SP600125 that caused toxicity below the $\mathrm{IC}_{5}$ were used: LLC, $5 \mu \mathrm{M}$ and $4 \mathrm{~T} 1,3 \mu \mathrm{M}$. The results confirmed that SP600125 effectively suppressed JNK signaling at $5 \mu \mathrm{M}$ in LLC and $3 \mu \mathrm{M}$ in $4 \mathrm{~T} 1$ cells (Fig. 2B and C).

Blocking of JNK signaling sensitizes LLC and $4 T 1$ cells to $\gamma$-irradiation. LLC and 4T1 cells were treated with different concentrations of SP600125,5 and $3 \mu \mathrm{M}$, respectively (Fig. 3A). The DEF, the ratio of the radiation dose alone and in combination with SP600125, was calculated by clonogenic survival at $10 \%$ (D0.1). D0.1 was defined as the quotient of D0.1 RT/D0.1 $\mathrm{RT}+$ drug. A DEF $>1$ indicated radiosensitization. SP600125 showed a radiosensitizing effect on LLC and 4T1 cells with a DEF 0.1 of 1.11 and 1.21, respectively (Table I). Treatment with a dose of 8 Gy $\gamma$-irradiation following exposure to SP600125 caused significant decreases in the clonal formation of both cell lines (Fig. 3B; $\mathrm{P}<0.05$ ).
$J N K$ regulates $\gamma H 2 A X$ expression after $\gamma$-irradiation. Western blot results showed that $\gamma \mathrm{H} 2 \mathrm{AX}$ expression was significantly increased in the radiation treatment group compared with the level in the control and SP600125 treatment groups; however, it was decreased by SP600125 therapy in the LLC and 4T1 cell lines. Similarly, immunohistochemistry results showed that $\gamma \mathrm{H} 2 \mathrm{AX}$ focus formation was significantly increased in the radiation treatment group compared with that noted in the control and SP600125 treatment groups, however, it was decreased by SP600125 treatment (Fig. 4A-D).

Inhibition of JNK signaling induces apoptosis after $\gamma$-irradiation. Western blot results showed that the levels of BAX, P-Bcl-2, cleaved caspase-3 and cleaved PARP were significantly increased in the radiation treatment group compared with these levels in the control and SP600125 treatment groups. Moreover, this effect was enhanced by SP600125 treatment in the LLC and 4T1 cells (Fig. 5).

Combination of JNK inhibitor with fractionated irradiation delays LLC tumor growth and promotes apoptosis in vivo. In the toxicity study on SP600125, tumor growth was not significantly delayed in the $5 \mu \mathrm{M}$ SP600125 treatment group compared with the vehicle group in the hind limb LLC tumor model, while there was significant delay in tumor growth in the SP600125 treatment group after fractionated radiotherapy in the radiosensitivity study (Fig. 6A and B). Tumor regrowth initiated $\sim 6$ days after $\mathrm{RT}$ was markedly reduced in the SP600125-irradiated tumors. Imaging of tumor size revealed that combined treatment with radiation and SP600125 produced a marked reduction in tumor volume at the end of the experiment (day 10) (Fig. 6C). Tumor weight was significantly decreased in the SP600125 treatment group compared with that noted in the untreated group after radiation (Fig. 6D; $\mathrm{P}<0.05)$. Immunohistochemistry results showed that the level of cleaved caspase- 3 was significantly increased in the group 
A

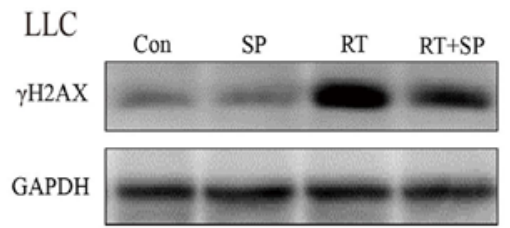

B

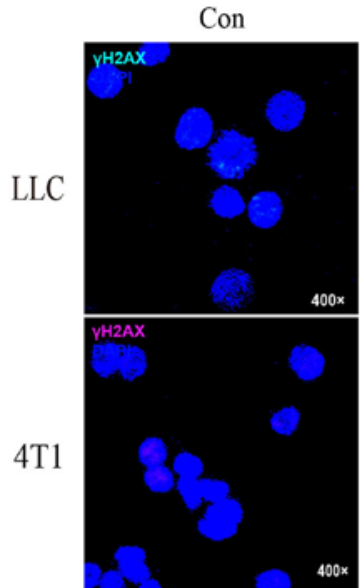

$\mathrm{C}$
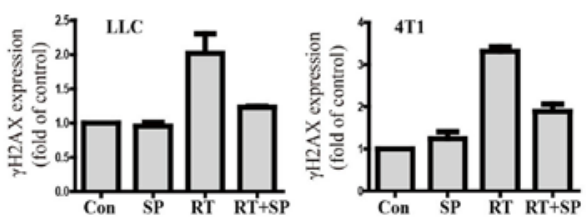

SP

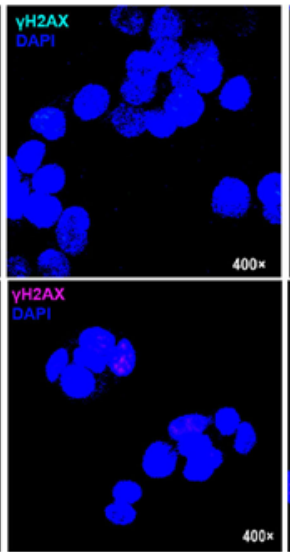

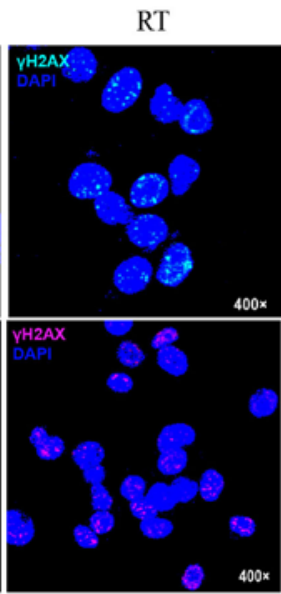

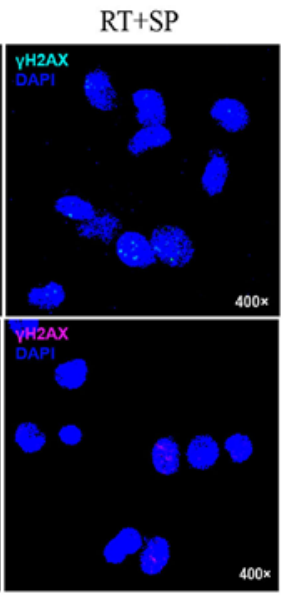

$\mathrm{D}$
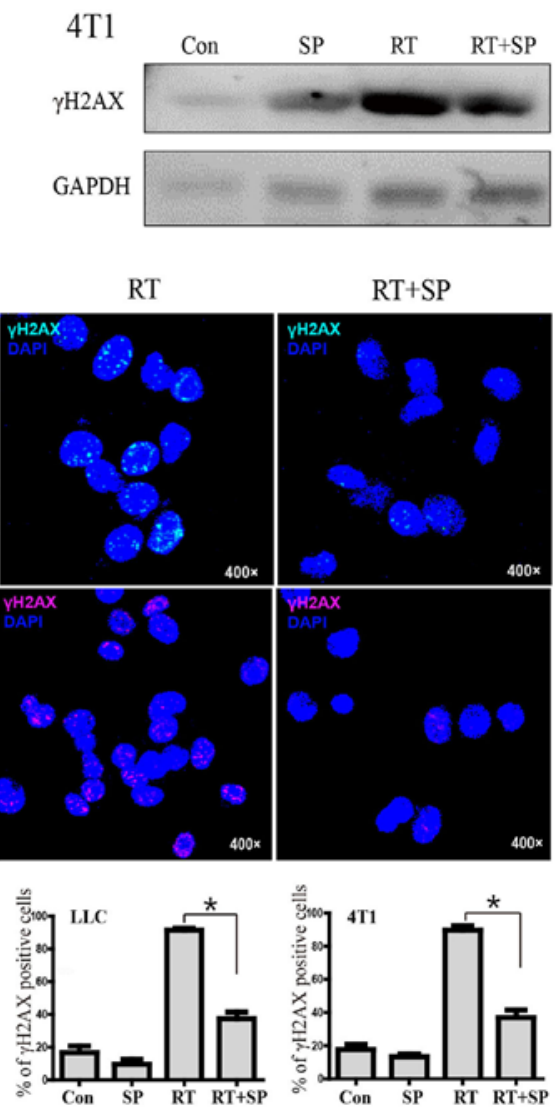

Figure 4. Blockade of JNK signaling reduces H2AX phosphorylation ( $\gamma \mathrm{H} 2 \mathrm{AX})$ after $\gamma$-irradiation. (A) The expression of $\gamma \mathrm{H} 2 \mathrm{AX}$ was measured via western blotting $2 \mathrm{~h}$ after $\gamma$-irradiation (8 Gy) in LLC (left) and 4T1 (right) cells. (B) Representative images of $\gamma \mathrm{H} 2 \mathrm{AX}$ detected $6 \mathrm{~h}$ after $\gamma$-irradiation (8 Gy) and immunostained with $\gamma \mathrm{H} 2 \mathrm{AX}$ antibody followed by Alexa 488 secondary antibody (green) or Alexa 568 secondary antibody (red). Nuclei were identified with DAPI (blue). (C) Histogram showing $\gamma \mathrm{H} 2 \mathrm{AX}$ expression levels compared to each treatment in both cell lines. (D) Percentage of $\gamma \mathrm{H} 2 \mathrm{AX}$ foci-positive cells was calculated in at least 50 cells. The images show positive cells containing $>5$ foci. Bars indicate \pm SD; columns, mean; SP, SP600125 (5 $\mu \mathrm{M}$ for LLC; $3 \mu \mathrm{M}$ for 4T1); RT, radiation ( $\gamma$-irradiation $8 \mathrm{~Gy}) ;{ }^{*} \mathrm{P}<0.05$ vs. the RT.

A

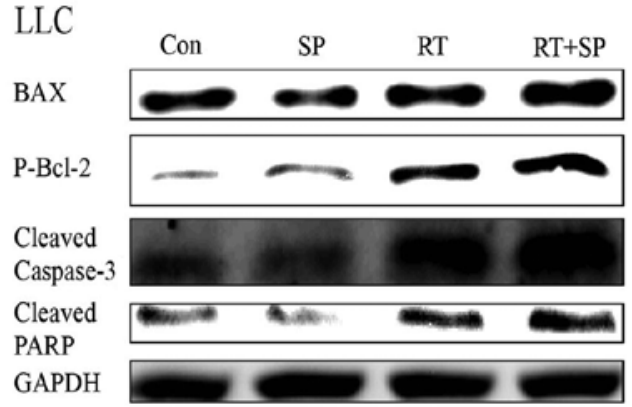

B

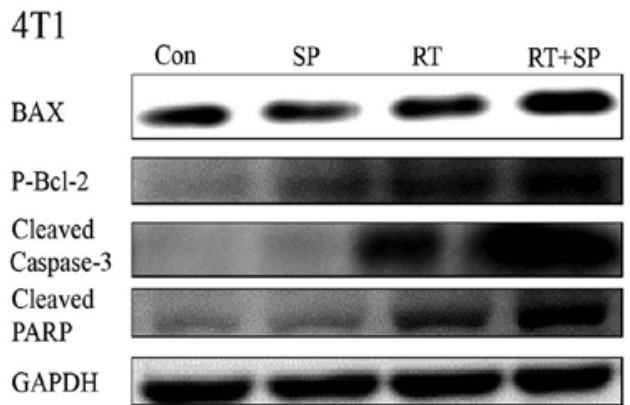

Figure 5. Inhibition of JNK signaling induces apoptosis after $\gamma$-irradiation. The expression of apoptosis-related proteins was confirmed by western blot analysis $24 \mathrm{~h}$ after $\gamma$-irradiation in the (A) LLC and (B) 4T1 cells. Bars indicate \pm SD; columns, mean; SP, SP600125 (5 $\mu \mathrm{M}$ for LLC; $3 \mu \mathrm{M}$ for 4T1); RT, radiation $(\gamma$-irradiation $8 \mathrm{~Gy})$.

receiving combined SP600125 and radiation compared with the other groups (Fig. 6E).

\section{Discussion}

It has been suggested that various drugs enhance the radiosensitivity of metastatic brain tumor cells. Thus, more and more research has focused on radiosensitizers to overcome the limitations of radiation therapy. The most common sources of brain metastases are lung (39\%) and breast cancer $(10-16 \%)(13,14)$. Therefore, lung and breast cancer cell lines were used in the present study.

Reducing the ability to repair DNA and increasing the effects of radiotherapy are the objectives of a prolonged endeavor in translational radiation research (15). Moreover, $\mathrm{H} 2 \mathrm{AX}$ plays a key role in the DNA repair system. $\gamma \mathrm{H} 2 \mathrm{AX}$, which is the phosphorylated form of $\mathrm{H} 2 \mathrm{AX}$, is recruited to various repair-related proteins such as p53 binding protein 1 

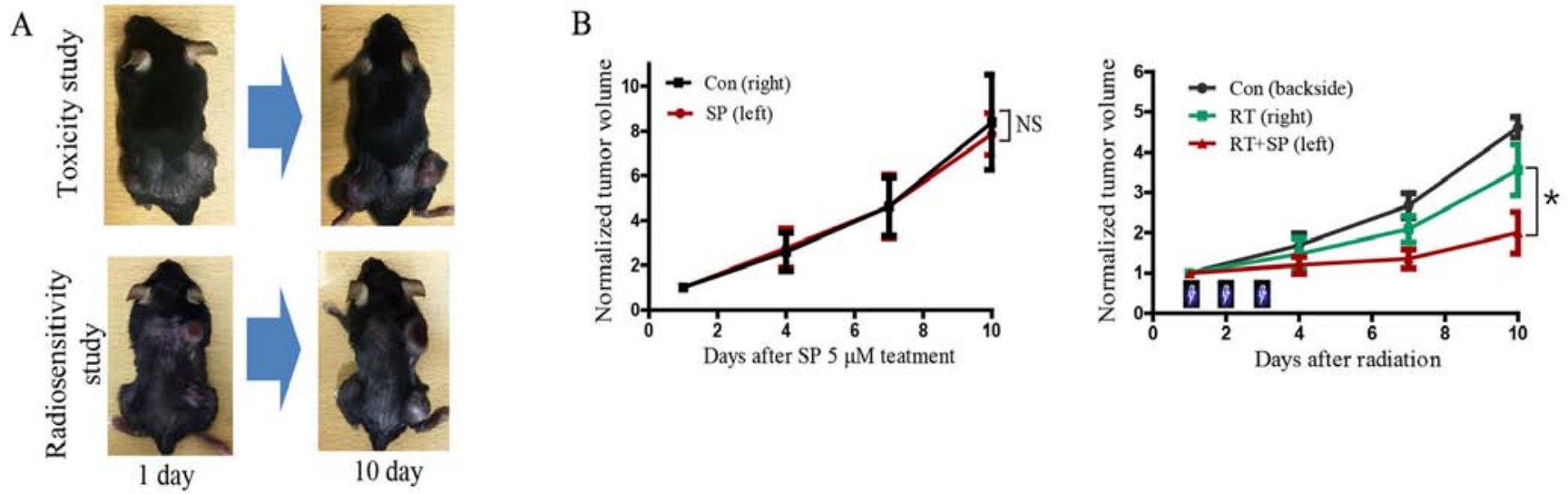

$\mathrm{C}$
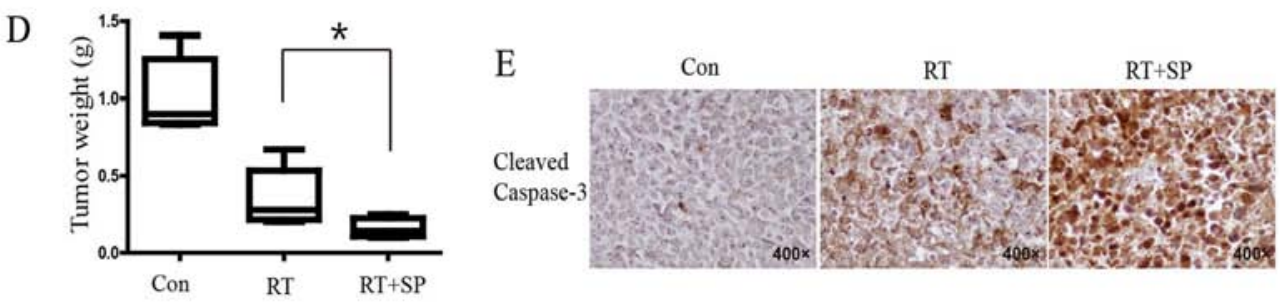

Figure 6. Combination of JNK inhibitor with fractionated irradiation delays LLC tumor growth and promotes apoptosis in vivo. (A) Images of mice at the beginning of treatment and at the end of the experiment. Top images, toxicity study of SP600125; lower images, tumor radiosensitivity study. (B) The data (left) show that SP600125 is not toxic at a concentration of $5 \mu \mathrm{M}$ in vivo. Normalized tumor growth curves (right) for mice after radiation at $3 \mathrm{x} 3.5$ Gy daily and/or treated with SP600125. (C) Images of tumors at the termination of the radiosensitivity study. (D) Weights of individual tumors at the end of the study. (E) Immunohistochemistry staining results for cleaved caspase-3 in tumors. Bars indicate \pm SD; columns, mean; SP, SP600125 (5 $\mu \mathrm{M}$ ); RT, radiation; NS = not significant; ${ }^{*} \mathrm{P}<0.05$ vs. the RT.

(53BP1), breast cancer associated gene 1 (BRCA1), Rad50 and NBS1 after DSB $(16,17)$. It is reported that ATM and Rad3-related protein (ATR), ataxia telangiectasia mutated (ATM) and DNA-dependent protein kinase (DNA-PK) play important roles in the phosphorylation of H2AX (18-20). Moreover, Lu et al demonstrated that JNK can phosphorylate $\mathrm{H} 2 \mathrm{AX}$ in vitro (4).

JNK protein is a subfamily of the mitogen activated protein kinase (MAPK) superfamily (21). JNK consists of 10 isoforms, which are derived from JNK1, JNK2 and JNK3 (22). JNK phosphorylation modifies the activity of a number of proteins that reside in the mitochondria or act in the nucleus, JNK1 and JNK2 are found in all cell and tissue types, while JNK3 is mainly found in the brain, but also in the heart and testes (2). JNK is involved in cellular differentiation and proliferation, neurodegeneration, inflammatory conditions and apoptosis mediated by activation protein-1 (AP-1), RANTES, IL-8 and GM-CSF (23). Yue et al reported that inhibition of JNK activity can enhance the radiosensitivity of vestibular schwannoma cells (5).

In the present study, P-JNK was significantly increased after radiation in the LLC and $4 \mathrm{Tl}$ cell lines. P-JNK may play a vital role in H2AX phosphorylation. Unfortunately, P-JNK is not dose-dependently increased after radiation. The JNK inhibitor SP600125 is a small-molecule inhibitor that competes with ATP to inhibit the phosphorylation of c-Jun $(6,24)$. Our results showed that SP600125 has dose-dependent cytotoxic effect on the LLC and 4T1 cell lines. Previous studies have demonstrated that phosphorylation of $\mathrm{H} 2 \mathrm{AX}$ is timedependently increased after radiation (25). Thus, $\gamma \mathrm{H} 2 \mathrm{AX}$ was measured $2 \mathrm{~h}$ after irradiation by western blotting and $6 \mathrm{~h}$ after irradiation by immunofluorescence staining. Our results showed that $\gamma \mathrm{H} 2 \mathrm{AX}$ expression was significantly increased after radiation. However, it was decreased by SP600125 treatment in both the LLC and 4T1 cell lines. These data suggest that phosphorylation of $\mathrm{H} 2 \mathrm{AX}$ was suppressed by inhibition of JNK, and to the best of our knowledge, this is the first study that $\gamma \mathrm{H} 2 \mathrm{AX}$ is inhibited by a JNK inhibitor after radiation in LLC and 4T1 cell lines.

Radiosensitivity was measured by clonogenic survival, the golden standard for measurement of radiation sensitization. Our clonogenic assay results showed that the necessary dose of radiation was significantly decreased in the SP600125-treated group. This suggests that SP600125 increased the radiosensitivity of the LLC and $4 \mathrm{~T} 1$ cells. It has been reported that P-JNK levels are increased after radiation and are associated with apoptosis $(26,27)$. Our results suggest that increased P-JNK levels may contribute to increased phosphorylation of H2AX after radiation. However, the mechanisms of action of the JNK inhibitor in enhancing radiosensitivity has not yet been fully understood.

Previous studies have demonstrated that reactive oxygen species (ROS) levels are increased in the early (within msec) and late (2-8 days) stages after radiation (28). ROS are formed as a natural byproduct of oxygen metabolism and play a vital function in cell signaling and homeostasis. ROS generally include the hydroxyl radicals $(\mathrm{OH})$, superoxide anion $\left(\mathrm{O}_{2}{ }^{-}\right)$ and hydrogen peroxide $\left(\mathrm{H}_{2} \mathrm{O}_{2}\right)$, which are able to damage omnifarious molecular targets including proteins, DNA and lipids $(29,30)$. Yue et al demonstrated that radiation enhanced ROS generation, and ROS significantly increased in the radiation and SP600125 treatment group compared with the only 
radiation group in vestibular schwannoma cells $(5,12)$. It has been reported that mitochondrial and JNK pathways play vital functions in ROS-induced cellular apoptosis $(29,31)$. The mitochondrial pathway is one of the typical apoptosis pathways. BAX interacts with Bcl-2 and promotes the entry of cytochrome $c$ into the cytoplasm. Cytochrome $c$ and caspase-9 combine to form an apoptotic body. Finally, caspase-3 is activated, and the combination of activated caspase- 3 and an apoptotic substrate leads to apoptosis $(30,32,33)$. PARP is one of the enzymatic substrates of caspase- 3 in the nucleus. It plays an important role in DNA replication, transcription, maintenance and adjustment of chromosomal structure, stabilization of the genome and cellular apoptosis $(34,35)$. PARP can be degraded by caspase- 3 and other caspases. Thus, proteolysis is considered to be an early molecular marker of apoptosis (36). Our results showed that expression of BAX, P-Bcl-2, cleaved caspase-3 and cleaved PARP were increased in the SP600125-treated group compared with these levels in the untreated group after radiation. These findings suggest that SP600125 has a radiosensitizing effect in LLC and 4T1 cells, and increases apoptosis after radiation.

Moreover, our in vivo study results showed that there was no significant change after $5 \mu \mathrm{M}$ SP600125 treatment compared with vehicle treatment in the bilateral flank LLC tumor model. However, tumor growth was significantly delayed in the SP600125 treatment group after fractionated radiation therapy in the backside of upper thoracic area and both flanks of the mouse tumor model. These results suggest that SP600125 has antitumor activity and suppressed tumor growth via inhibition of $\gamma \mathrm{H} 2 \mathrm{AX}$ and DNA repair in the mouse model.

Normal brain cells, including neurons, are sensitive to radiation; thus, the appropriate dose is limited in radiotherapy. Therefore, a radiosensitizer provides a new method for radiotherapy. JNK inhibitor increases radiosensitivity in vestibular schwannoma and lung and breast cancer cells. Further studies are needed to evaluate radiosensitivity in other cells.

In conclusion, blocking of JNK signaling decreased $\gamma \mathrm{H} 2 \mathrm{AX}$ expression and increased apoptosis in lung and breast cancer cells after radiotherapy. JNK plays an important role during radiotherapy as a radiosensitizer via suppression of the DNA repair system. In future, JNK inhibitors may be used as alternative radiosensitizers to improve the outcomes of radiotherapy for metastatic brain tumors.

\section{References}

1. Davis RJ: Signal transduction by the JNK group of MAP kinases Cell 103: 239-252, 2000.

2. Bode AM and Dong Z: The functional contrariety of JNK. Mol Carcinog 46: 591-598, 2007.

3. Weston CR and Davis RJ: The JNK signal transduction pathway. Curr Opin Cell Biol 19: 142-149, 2007.

4. Lu C, Zhu F, Cho YY, Tang F, Zykova T, Ma WY, Bode AM and Dong Z: Cell apoptosis: Requirement of H2AX in DNA ladder formation, but not for the activation of caspase-3. Mol Cell 23: 121-132, 2006.

5. Yue WY, Clark JJ, Telisak M and Hansen MR: Inhibition of c-Jun $\mathrm{N}$-terminal kinase activity enhances vestibular schwannoma cell sensitivity to gamma irradiation. Neurosurgery 73: 506-516, 2013.

6. Bennett BL, Sasaki DT, Murray BW, O'Leary EC, Sakata ST, Xu W, Leisten JC, Motiwala A, Pierce S, Satoh Y, et al: SP600125, an anthrapyrazolone inhibitor of Jun N-terminal kinase. Proc Natl Acad Sci USA 98: 13681-13686, 2001.
7. Guan QH, Pei DS, Zhang QG, Hao ZB, Xu TL and Zhang GY: The neuroprotective action of SP600125, a new inhibitor of JNK, on transient brain ischemia/reperfusion-induced neuronal death in rat hippocampal CA1 via nuclear and non-nuclear pathways. Brain Res 1035: 51-59, 2005.

8. Li S, Li C, Ryu HH, Lim SH, Jang WY and Jung S: Bacitracin inhibits the migration of U87-MG glioma cells via interferences of the integrin outside-in signaling pathway. J Korean Neurosurg Soc 59: 106-116, 2016.

9. Jin SG, Ryu HH, Li SY, Li CH, Lim SH, Jang WY and Jung S: Nogo-A inhibits the migration and invasion of human malignant glioma U87MG cells. Oncol Rep 35: 3395-3402, 2016.

10. Wen M, Jung S, Moon KS, Jiang SN, Li SY and Min JJ: Targeting orthotopic glioma in mice with genetically engineered Salmonella typhimurium. J Korean Neurosurg Soc 55: 131-135, 2014.

11. Wang W, Shi L, Xie Y, Ma C, Li W, Su X, Huang S, Chen R, Zhu Z, Mao Z, et al: SP600125, a new JNK inhibitor, protects dopaminergic neurons in the MPTP model of Parkinson's disease. Neurosci Res 48: 195-202, 2004.

12. Yue WY, Clark JJ, Fernando A, Domann F and Hansen MR: Contribution of persistent C-Jun N-terminal kinase activity to the survival of human vestibular schwannoma cells by suppression of accumulation of mitochondrial superoxides. Neuro Oncol 13: 961-973, 2011.

13. Lin NU, Bellon JR and Winer EP: CNS metastases in breast cancer. J Clin Oncol 22: 3608-3617, 2004.

14. Shaw MG and Ball DL: Treatment of brain metastases in lung cancer: Strategies to avoid/reduce late complications of whole brain radiation therapy. Curr Treat Options Oncol 14: 553-567, 2013.

15. Jackson SP and Bartek J: The DNA-damage response in human biology and disease. Nature 461: 1071-1078, 2009.

16. Fernandez-Capetillo O, Chen HT, Celeste A, Ward I, Romanienko PJ, Morales JC, Naka K, Xia Z, Camerini-Otero RD, Motoyama $\mathrm{N}$, et al: DNA damage-induced $\mathrm{G}_{2}-\mathrm{M}$ checkpoint activation by histone H2AX and 53BP1. Nat Cell Biol 4: 993-997, 2002.

17. Paull TT, Rogakou EP, Yamazaki V, Kirchgessner CU, Gellert M and Bonner WM: A critical role for histone $\mathrm{H} 2 \mathrm{AX}$ in recruitment of repair factors to nuclear foci after DNA damage. Curr Biol 10: 886-895, 2000

18. Burma S, Chen BP, Murphy M, Kurimasa A and Chen DJ: ATM phosphorylates histone $\mathrm{H} 2 \mathrm{AX}$ in response to DNA double-strand breaks. J Biol Chem 276: 42462-42467, 2001.

19. Wang H, Wang M, Wang H, Böcker W and Iliakis G: Complex H2AX phosphorylation patterns by multiple kinases including ATM and DNA-PK in human cells exposed to ionizing radiation and treated with kinase inhibitors. J Cell Physiol 202: 492-502, 2005.

20. Ward IM and Chen J: Histone H2AX is phosphorylated in an ATR-dependent manner in response to replicational stress. J Biol Chem 276: 47759-47762, 2001.

21. Hibi M, Lin A, Smeal T, Minden A and Karin M: Identification of an oncoprotein- and UV-responsive protein kinase that binds and potentiates the c-Jun activation domain. Genes Dev 7: 2135-2148, 1993.

22. Waetzig V and Herdegen T: Context-specific inhibition of JNKs: Overcoming the dilemma of protection and damage. Trends Pharmacol Sci 26: 455-461, 2005.

23. Oltmanns U, Issa R, Sukkar MB, John M and Chung KF: Role of c-jun N-terminal kinase in the induced release of GM-CSF, RANTES and IL-8 from human airway smooth muscle cells. Br J Pharmacol 139: 1228-1234, 2003.

24. Barr RK, Kendrick TS and Bogoyevitch MA: Identification of the critical features of a small peptide inhibitor of JNK activity. J Biol Chem 277: 10987-10997, 2002.

25. An J, Huang YC, Xu QZ, Zhou LJ, Shang ZF, Huang B, Wang Y, Liu XD, Wu DC and Zhou PK: DNA-PKcs plays a dominant role in the regulation of $\mathrm{H} 2 \mathrm{AX}$ phosphorylation in response to DNA damage and cell cycle progression. BMC Mol Biol 11: 18, 2010.

26. Kim CH, Won M, Choi CH, Ahn J, Kim BK, Song KB, Kang CM and Chung KS: Increase of RhoB in gamma-radiation-induced apoptosis is regulated by c-Jun N-terminal kinase in Jurkat $\mathrm{T}$ cells. Biochem Biophys Res Commun 391: 1182-1186, 2010.

27. Han Y, Wang Y, Xu HT, Yang LH, Wei Q, Liu Y, Zhang Y, Zhao Y, Dai SD, Miao Y, et al: X-radiation induces non-smallcell lung cancer apoptosis by upregulation of Axin expression. Int J Radiat Oncol Biol Phys 75: 518-526, 2009. 
28. Gao Z, Sarsour EH, Kalen AL, Li L, Kumar MG and Goswami PC: Late ROS accumulation and radiosensitivity in SOD1-overexpressing human glioma cells. Free Radic Biol Med 45: 1501-1509, 2008.

29. Shen HM and Liu ZG: JNK signaling pathway is a key modulator in cell death mediated by reactive oxygen and nitrogen species. Free Radic Biol Med 40: 928-939, 2006.

30. Jena NR: DNA damage by reactive species: Mechanisms, mutation and repair. J Biosci 37: 503-517, 2012.

31. Yu CC, Ko FY, Yu CS, Lin CC, Huang YP, Yang JS, Lin JP and Chung JG: Norcantharidin triggers cell death and DNA damage through S-phase arrest and ROS-modulated apoptotic pathways in TSGH 8301 human urinary bladder carcinoma cells. Int J Oncol 41: 1050-1060, 2012.

32. Zeng H, Kong X, Peng H, Chen Y, Cai S, Luo H and Chen P: Apoptosis and $\mathrm{Bcl}-2$ family proteins, taken to chronic obstructive pulmonary disease. Eur Rev Med Pharmacol Sci 16: 711-727, 2012.
33. Tang S, Hu J, Meng Q, Dong X, Wang K, Qi Y, Chu C, Zhang X and Hou L: Daidzein induced apoptosis via down-regulation of $\mathrm{Bcl}-2 / \mathrm{Bax}$ and triggering of the mitochondrial pathway in BGC-823 cells. Cell Biochem Biophys 65: 197-202, 2013.

34. Miwa M and Masutani M: PolyADP-ribosylation and cancer. Cancer Sci 98: 1528-1535, 2007.

35. Wei L, Nakajima S, Hsieh CL, Kanno S, Masutani M, Levine AS, Yasui A and Lan L: Damage response of XRCC1 at sites of DNA single strand breaks is regulated by phosphorylation and ubiquitylation after degradation of poly(ADP-ribose). J Cell Sci 126: 4414-4423, 2013

36. Germain M, Affar EB, D'Amours D, Dixit VM, Salvesen GS and Poirier GG: Cleavage of automodified poly(ADP-ribose) polymerase during apoptosis. Evidence for involvement of caspase-7. J Biol Chem 274: 28379-28384, 1999. 